\section{OPEN ACCESS}

Edited by:

Helioswilton Sales-Campos, Universidade Federal de Goiás, Brazil

Reviewed by:

Fillipe Luiz Rosa do Carmo,

INRA UMR1253 Science and Technologie du Lait \& de l'œuf, France Gislane Lelis Vilela de Oliveira, São Paulo State University, Brazil

${ }^{*}$ Correspondence: Cecilia I. Muglia cmugliaenator@gmail.com

${ }^{\dagger}$ These authors share senior authorship

Specialty section: This article was submitted to Inflammation Pharmacology, a section of the journal Frontiers in Pharmacology

Received: 24 January 2021 Accepted: 04 March 2021 Published: 15 April 2021

Citation:

Curciarello R, Canziani KE, Salto I, Barbiera Romero E, Rocca A, Doldan I, Peton E, Brayer S, Sambuelli AM, Goncalves S, Tirado P, Correa GJ, Yantorno M, Garbi L, Docena GH, Serradell MdIÁ and Muglia Cl (2021) Probiotic Lactobacilli Isolated from Kefir Promote Down-Regulation of Inflammatory Lamina Propria T Cells

from Patients with Active IBD.

Front. Pharmacol. 12:658026. doi: 10.3389/fphar.2021.658026

\title{
Probiotic Lactobacilli Isolated from Kefir Promote Down-Regulation of Inflammatory Lamina Propria T Cells from Patients with Active IBD
}

\begin{abstract}
Renata Curciarello ${ }^{1}$, Karina E. Canziani ${ }^{1}$, lleana Salto ${ }^{1}$, Emanuel Barbiera Romero ${ }^{1}$, Andrés Rocca ${ }^{2}$, Ivan Doldan ${ }^{2}$, Emmanuel Peton ${ }^{3}$, Santiago Brayer ${ }^{3}$, Alicia M. Sambuelli ${ }^{4}$, Silvina Goncalves ${ }^{4}$, Pablo Tirado ${ }^{4}$, Gustavo J. Correa ${ }^{5}$, Martín Yantorno ${ }^{5}$, Laura Garbi ${ }^{5}$, Guillermo H. Docena ${ }^{1}$, María de los Ángeles Serradell ${ }^{6+}$ and Cecilia I. Muglia ${ }^{1+*}$
\end{abstract}

${ }^{1}$ Instituto de Estudios Inmunológicos y Fisiopatológicos (IIFP), CONICET-Departamento de Ciencias Biológicas, Facultad de Ciencias Exactas, Universidad Nacional de La Plata, Asociado CIC PBA, La Plata, Argentina, ${ }^{2}$ Unidad Endoscopía, Hospital de Gastroenterología Dr. Carlos Bonorino Udaondo, Ciudad Autónoma de Buenos Aires, Argentina, ${ }^{3}$ Unidad de Proctología, Departamento de Cirugía, Hospital de Gastroenterología Dr. Carlos Bonorino Udaondo, Ciudad Autónoma de Buenos Aires, Argentina, ${ }^{4}$ Sección de Enfermedades Inflamatorias Del Intestino, Hospital de Gastroenterología Dr. Carlos Bonorino Udaondo, Ciudad Autónoma de Buenos Aires, Argentina, ${ }^{5}$ Área de Enfermedad Inflamatoria Intestinal, Sala de Endoscopía, Servicio de Gastroenterología, Hospital Interzonal General de Agudos General San Martín, La Plata, Argentina, ${ }^{6}$ Cátedra de Microbiología, Departamento de Ciencias Biológicas, Facultad de Ciencias Exactas, Universidad Nacional de La Plata, La Plata, Argentina

Ulcerative colitis and Crohn's disease, the two main forms of inflammatory bowel disease (IBD), are immunologically mediated disorders. Several therapies are focused on activated T cells as key targets. Although Lactobacillus kefiri has shown anti-inflammatory effects in animal models, few studies were done using human mucosal T cells. The aim of this work was to investigate the immunomodulatory effects of this bacterium on intestinal $T$ cells from patients with active IBD. Mucosal biopsies and surgical samples from IBD adult patients $(n=19)$ or healthy donors (HC; $n=5)$ were used. Lamina propria mononuclear cells were isolated by enzymatic tissue digestion, and entero-adhesive Escherichia coli-specific lamina propria T cells (LPTC) were expanded. The immunomodulatory properties of $L$. kefiri CIDCA 8348 strain were evaluated on biopsies and on anti-CD3/CD28-activated LPTC. Secreted cytokines were quantified by ELISA, and cell proliferation and viability were assessed by flow cytometry. We found that $L$. kefiri reduced spontaneous release of IL-6 and IL-8 from inflamed biopsies ex vivo. Activated LPTC from IBD patients showed low proliferative rates and reduced secretion of TNF- $\alpha$, IL-6, IFN- $\gamma$ and IL-13 in the presence of L. kefiri. In addition, $L$. kefiri induced an increased frequency of CD4 ${ }^{+} F O X P 3^{+}$LPTC along with high levels of $\mathrm{IL}-10$. This is the first report showing an immunomodulatory effect of $L$. kefiri CIDCA 8348 on human intestinal cells from IBD patients. Understanding the mechanisms of interaction between probiotics and immune mucosal cells may open new avenues for treatment and prevention of IBD.

Keywords: ulcerative colitis, Crohn's disease, probiotics, immunomodulation, mucosal samples, Lactobacillus kefiri CIDCA 8348 


\section{INTRODUCTION}

Inflammatory bowel disease (IBD) comprises a complex group of chronic relapsing diseases among which the most conspicuous are ulcerative colitis (UC) and Crohn's disease (CD). Patients with IBD suffer chronic inflammation of the bowel mucosa that may affect the mucosal layer (UC) or the whole bowel wall (CD) (Torres et al., 2017; Ungaro et al., 2017). The etiology of these diseases is largely unknown, although factors such as diet, certain genes related to the sensing of luminal microbes, secretion of antimicrobial peptides and autophagy have shown to be associated (J. S. Lee et al., 2021; Kaser and Blumberg 2011). Changes in microbiota composition are typically observed in IBD patients. These findings, along with results obtained from animal models, including the fact that germ free mice do not develop experimental colitis, highlight the impact of the microbiota composition in the pathogenesis of these disorders (Onderdonk et al., 1977).

Metagenomic strategies have revealed an altered gut microbial composition in IBD patients compared to healthy subjects, known as dysbiosis (Liu et al., 2020). IBD patients usually present a reduced bacterial diversity, with low levels of Firmicutes and Bacteroidetes and increased levels of facultative anaerobic Proteobacteria and Bacilli. However, the implication of these findings for pathogenesis is not clear. Also, persistent bacterial infection by enteric bacteria, such as adherentinvasive Escherichia coli, has been observed (Abdelhalim et al., 2020; J. G. Lee et al., 2019). Intensive research is being carried out to determine whether changes in microbiota are causative or a consequence of the chronic inflammation observed in IBD. Inflammation probably comes from a sum of effects: an increased amount of mucosal associated bacteria, along with high intestinal permeability also present in these patients suggest that bacteria could penetrate the epithelial barrier, thus contributing to inflammation. These microorganisms in turn may promote the release of pro-inflammatory factors such as TNF- $\alpha$, which boost inflammation (Shawki and McCole, 2017). Altered trans-cellular and para-cellular permeability have been described in IBD, evidenced by the presence of intracellular bacteria inside epithelial cells, and by modified tight junction protein expression and increased myosin light chain kinase (MLCK) activity (Yu Chia-Hui, 2018). Moreover, gut permeability can be influenced by changes in metabolites produced by the microbiota (Schlegel et al., 2020; J. S. Lee et al., 2021). The healthy gut microbiome produces bioactive metabolites, including short chain fatty acids (SCFA), which contribute to intestinal homeostasis and epithelial cell nutrition (Postler et al., 2017). Diminished levels of these molecules could favor an impaired barrier function and inflammatory environment.

The role of $\mathrm{CD}^{+} \mathrm{T}$ lymphocytes is critical in IBD. In CD, IL12 signaling induces the differentiation of $\mathrm{CD} 4^{+} \mathrm{T}$ cells into IFN$\gamma$ secreting cells, while IL-23 contributes to the differentiation of $\mathrm{CD} 4{ }^{+} \mathrm{T}$ cells into Th1 and Th17, thus increasing IFN- $\gamma$ and IL-17 secretion, respectively. IL-6, IL-23 and TGF- $\beta$ also participate in $\mathrm{CD}$ pathogenesis. In $\mathrm{UC}, \mathrm{CD} 4^{+} \mathrm{T}$ lymphocytes also secrete IL-4 and IL-13, which contribute to tissue damage (Zenewicz et al., 2009). In addition, activated $\mathrm{CD}^{+} \mathrm{T}$ cells have increased proliferation rate and are resistant to apoptosis in these pathologies (Schmitt et al., 2019). Anti-TNF- $\alpha$ therapies target these activated $\mathrm{T}$ cells, inducing $\mathrm{T}$ cell apoptosis, but unfortunately, many patients do not respond or become refractory within years of treatment, and require surgery (Yanai and Hanauer, 2011). Consequently, great effort is being made to develop new therapies for IBD, aimed to modulate T cell response.

Probiotics were defined several years ago as "live microorganisms that confer a health benefit to the host when administered in adequate amounts" (Food and Agriculture Organization and of the United Nations/World Health Organization, 2002). Recently, the term "postbiotic" has come to be used and refers to the functional bioactive compounds generated during microbial fermentation processes, including extracellular polysaccharides, (SCFA) and different microbial cell components, which can have beneficial effects on host health (Wegh et al., 2019). Probiotics have been studied as having beneficial properties in murine and rat models of colitis. Different strains of Bifidobacterium, E. coli and Lactobacillus have shown anti-inflammatory effects (Jakubczyk et al., 2020). Even though data from probiotics treatment in patients are controversial, evidence of their usefulness in combination with pharmacological treatments is arising (Shanahan and Quigley, 2014; Qiao et al., 2016; Fan et al., 2019; Ahn et al., 2020). Nevertheless, basic studies regarding their effect on IBD lamina propria cells are scarce. Kefir is an ancient product traditionally obtained by fermentation of milk with kefir grains, and many health-promoting properties have been associated with its consumption (Farag et al., 2020). Kefir grains are composed of different species of bacteria and yeasts that live symbiotically in a complex matrix constituted by proteins and polysaccharides (Bengoa et al., 2019). Lactobacillus kefiri is one of the most important lactobacilli retrieved from kefir, with reported quantification of around $10^{8}$ bacteria/mL in fermented milk (Garrote et al., 2005). Most L. kefiri strains are safe for consumption and resistant to the harsh conditions of the gastrointestinal tract. Moreover, different beneficial effects, including immunomodulation and prevention of metabolic disorders, have been reported for these species (Slattery et al., 2019). In particular, the kefirisolated strain L. kefiri CIDCA 8348 has shown to be sensitive to several antibiotics, to lack virulence factors, and to be safe for oral consumption in mice (Carasi et al., 2014). Noteworthy, mice orally treated with this strain showed reduced expression of proinflammatory molecules and an up-regulation of antiinflammatory mediators, as well as secretory $\operatorname{IgA}$ and mucins in the gut (Carasi et al., 2015). Moreover, it was reported that administration of L. kefiri CIDCA 8348 prevents the deleterious effects of a fructose-rich diet in a murine model, exerting an anti-inflammatory activity in the adipose tissue (Zubiría et al., 2017).

In this work, we studied the immunomodulatory properties of L. kefiri CIDCA 8348 on $\mathrm{CD}^{+} \mathrm{T}$ lymphocytes from the lamina propria of IBD patients. This Lactobacillus strain diminishes the proliferation of these cells and the secretion of pro-inflammatory cytokines through an NF- $\kappa \mathrm{B}$ dependent pathway. 
TABLE 1 | Clinical features of healthy control $(\mathrm{HC})$ and IBD patient groups.

\begin{tabular}{|c|c|c|c|}
\hline & \multirow[t]{2}{*}{ HC group } & \multicolumn{2}{|c|}{ IBD group } \\
\hline & & UC & CD \\
\hline Number of patients & 5 & 11 & 8 \\
\hline Sex $(n=$ patients $)$ & Female $=3$, Male $=2$ & Female $=6$, Male $=5$ & Female $=7$, Male $=1$ \\
\hline Age of the patients [average (range)] & $51(23-73)$ y & $39(18-64)$ y & $38(21-59)$ y \\
\hline Site of sampling $\left(n=\right.$ patients) ${ }^{\mathrm{a}}$ & $\begin{array}{l}\text { Left colon (2) } \\
\text { rectum (3) }\end{array}$ & $\begin{array}{l}\text { Cecum (1), right (2), left (4), transverse (2), } \\
\text { sigmoid (3), rectum (8) }\end{array}$ & $\begin{array}{l}\text { Cecum (2), right (3), left (2), transverse (1), } \\
\text { rectum ( } 2 \text { ) }\end{array}$ \\
\hline $\begin{array}{l}\text { Endoscopic activity at sampling time } \\
\text { ( } n=\text { patients) }\end{array}$ & No activity $=5$ & mild $=6$, moderate $=3$ severe $=2$ & No activity $=1^{\mathrm{b}}$, mild $=1$, moderate $=4$ severe $=2$ \\
\hline 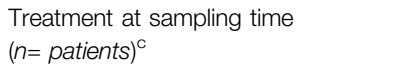 & Not applicable & $\begin{array}{l}\text { Adalimumab (1) corticosteroids (3) mesalazine } \\
\text { (9) azatioprine (4) }\end{array}$ & $\begin{array}{l}\text { Adalimumab (2) infliximab (1) corticosteroids (4) } \\
\text { mesalazine (3) azatioprine (2) }\end{array}$ \\
\hline
\end{tabular}

\section{MATERIALS AND METHODS}

\section{Patients and Tissue}

Surgical samples of colon or rectum from patients who underwent partial or total colectomy, and endoscopic colonic biopsies were taken from macroscopically inflamed mucosa of IBD adult patients affected by CD $(n=8)$ or UC $(n=11)$. The diagnosis was made according to clinical, endoscopic and histological criteria. The extent and location of the UC and $\mathrm{CD}$ were evaluated during colonoscopy. Clinical activity in UC was evaluated by total Mayo score (inactive $\leq 2$, mild activity three to five, moderate seven to nine, severe 10-12) and by HarveyBradshaw Index (Inactive score $<5$; mild activity $\geq 5$, moderate $\geq 7$, severe $\geq 16$ ) in CD (Table 1) (Peyrin-Biroulet et al., 2016). In addition, mucosal samples were collected endoscopically from the colon of adult subjects who were neither diagnosed with IBD nor any other inflammatory condition of the gut. Samples of healthy mucosa were obtained from surgical specimens of colorectal cancer partial colectomies. These specimens constituted the "healthy control patient" samples (HC, $n=5$ ). The local Ethics Committee (Comité de Etica en Investigaciones, Hospital de Gastroenterología Carlo B. Udaondo, Ciudad Autónoma de Buenos Aires, Res. 07-07-2016) approved the protocols and informed written consent was obtained from every patient.

\section{Bacteria and Conditioned Medium}

Lactobacillus kefiri CIDCA 8348 belonging to the collection of the Centro de Investigación y Desarrollo en Criotecnología de Alimentos (CIDCA, CONICET-UNLP-CIC, Argentina) was used. The strain was cultured in deMan-Rogosa-Sharpe (MRS) broth (Difco, Beauvais, France) at $37^{\circ} \mathrm{C}$ for $48 \mathrm{~h}$ in aerobic conditions. Bacteria were harvested, washed twice and finally resuspended in sterile phosphate-buffered saline (PBS) at $\mathrm{OD}^{550}=2.0$ (approx. $\left.1-2 \times 10^{8} \mathrm{cfu} / \mathrm{ml}\right)$. In order to prepare the L. kefiri-conditioned medium (CM), bacterial suspension at $\mathrm{OD}^{550}=0.1$ in Ultraculture medium was incubated for $24 \mathrm{~h}$ at $37^{\circ} \mathrm{C}$ and $5 \% \mathrm{CO}_{2}$. Then, bacteria were removed by centrifugation and supernatant was collected and stored at $-20^{\circ} \mathrm{C}$ until used. Entero-adhesive (EA) E. coli was grown in Luria Bertani Broth to $\mathrm{OD}^{550}=0.8$. Cells were then harvested by centrifugation and resuspended in sterile PBS. Extracts were sonicated for 10 pulses at $100 \%$ and centrifuged al $10.000 \times g$ for $15 \mathrm{~min}$. Protein concentration of the resulting solution was assayed by bicinchoninic acid assay (Pierce, Thermo Fisher Scientific, Rockford, IL, United States) and stored at $-20^{\circ} \mathrm{C}$. Prior to use, extracts were thawed and diluted in fresh medium to the desired concentration.

\section{Organ Cultures}

Endoscopic mucosal biopsies from control subjects $(n=5)$ or IBD patients $(n=13)$ were placed (one biopsy per well) in 24-well plates. Individual biopsies were cultured in $300 \mu \mathrm{L}$ of serum-free RPMI 1640 medium (Gibco, Thermo Fisher Scientific, Rockford, IL, United States), supplemented with $100 \mathrm{U} / \mathrm{mL}$ penicillin and $100 \mu \mathrm{g} / \mathrm{ml}$ streptomycin, and cultured at $37^{\circ} \mathrm{C}, 5 \% \mathrm{CO}_{2}$, with or without probiotics and/or $10 \mathrm{ng} / \mathrm{ml} \mathrm{TNF- \alpha .} \mathrm{After} 24 \mathrm{~h}$ of ex vivo culture, supernatants of mucosal biopsies were collected and stored at $-70^{\circ} \mathrm{C}$ until used.

\section{Lamina Propria Mononuclear Cell Isolation}

The mucosa layer of surgical pieces was mechanically separated from the full-thickness surgical specimen. The epithelial layer was removed with $1 \mathrm{mmol} / \mathrm{L}$ ethylenediaminetetraacetic acid (EDTA) and $1 \mathrm{mmol} / \mathrm{L}$ dithiothreitol (DTT) in $1 \mathrm{mM} \mathrm{HBSS}$ (Gibco, Thermo Fisher Scientific, Rockford, IL, United States). After stirring for $1 \mathrm{~h}$ at $37^{\circ} \mathrm{C}$, the supernatant was removed. The remaining tissue was minced with a scalpel and digested with type $1 \mathrm{~A}$ collagenase $(1 \mathrm{mg} / \mathrm{ml}$; Sigma-Aldrich, St. Louis, MO, United States) and DNAse (10 IU/ml, Roche, Thermo Fisher Scientific, Rockford, IL, United States) in RPMI-1640 medium containing $10 \%$ fetal bovine serum (FBS), $100 \mathrm{U} / \mathrm{mL}$ penicillin, and $100 \mu \mathrm{g} / \mathrm{ml}$ streptomycin for $2 \mathrm{~h}$ with stirring at $37^{\circ} \mathrm{C}$ and $5 \%$ $\mathrm{CO}_{2}$. Biopsies were washed three times in HBSS containing EDTA and DTT with stirring and then digested as described above. Cells were filtered through $40 \mu \mathrm{m}$ cell-strainers (Becton 
Dickinson, Franklin Lakes, NJ, United States) and washed with RPMI-1640 medium containing 10\% FBS, $100 \mathrm{U} / \mathrm{mL}$ penicillin, and $100 \mu \mathrm{g} / \mathrm{ml}$ streptomycin.

\section{Generation of Lamina Propria T-Cell Lines (LPTC)}

LPMC were washed and cultured in $300 \mu \mathrm{L}$ of serum-free Ultraculture medium (LONZA, Basel, Switzerland), supplemented with $2 \mathrm{mM}$ glutamine, $20 \mu \mathrm{M} \quad 2$ mercaptoethanol, and Antibiotic-Antimicotic (Gibco, Thermo Fisher Scientific, Rockford, IL, United States). In order to obtain specific LPTC, cells were stimulated with enteroadhesive E. coli extracts $(0.5 \mu \mathrm{g} / \mathrm{ml})$ at $37^{\circ} \mathrm{C}, 5 \% \mathrm{CO}_{2}$. Cultures without $E$. coli extract served as controls. Four days later, cells were treated with recombinant human (rh) IL-2 $(10 \mathrm{U} / \mathrm{mL}$, Preprotech, Rocky Hill, NJ, United States), rhIL-7 (10 ng/ml, Preprotech, NJ, United States) and rhIL-15 (10 ng/ml, Preprotech, Rocky Hill, NJ, United States) as reported by Rabinowitz et al. (2013). After 5 days, viable T cell blasts were enriched by Ficoll-Paque ${ }^{\text {тм }}$ (GE, Healthcare, Life Sciences, Danderyd, Sweden) gradient and then incubated in 96-well round-bottom cultures plates, in Ultraculture medium, supplemented with the cytokines mentioned above, $10 \%$ human $\mathrm{AB}^{+}$plasma and irradiated peripheral blood mononuclear cells PBMC $\left(1 \times 10^{5}\right)$ (Bohle et al., 2003). Cells were expanded in this same medium twice a week until enough cells were obtained, thus generating EA E. coli specific LPTC (from now on LPTC).

\section{LPTC Cultures and Proliferation Assays}

After expansion, LPTC were rested without further feeding for 10 days. Cells were washed, labeled with CFSE (Sigma-Aldrich, St. Louis, MO, United States) proliferation dye and stimulated with human anti-CD3 and anti-CD28 antibodies $(1 \mu \mathrm{g} / \mathrm{ml}$, eBioscience, San Diego, CA, United States), L. kefiri (2:1 bacteria:eukaryotic cell relation), L. kefiri conditioned media or combinations thereof. EA E. coli cultures were used as a positive control, also in a 2:1 relation to lymphocytes. Negative controls without the addition of stimuli were included. Assays were performed in serum-free AIMV medium (Thermo Fisher Scientific, Rockford, IL, United States). After 4 days, culture supernatants were harvested for cytokine evaluation and cells were stained with anti-CD4-APC (BD Pharmingen, San Diego, CA, United States), 7-AAD (BD Pharmingen, San Diego, CA, United States) and flow cytometry was performed using a FACS CALIBUR (BD, Franklin Lakes, NJ, United States). Flow cytometry data from two independent experiments from each patient was analyzed using FlowJo software (BD, Franklin Lakes, NJ, United States).

For intracellular FOXP3 staining, cells were stimulated as previously indicated. After 4 days cells were harvested and stained with anti-CD4-APC (BD Pharmingen, San Diego, CA, United States). Cells were then treated with BD Cytofix/ Cytoperm Fixation/Permeablization Kit (BD Pharmingen, San Diego, CA, United States) and stained with anti-FOXP3-PE (BD
Pharmingen, San Diego, CA, United States). Events were acquired with a FACS CALIBUR (BD, Franklin Lakes, NJ, United States). Lymphocytes were gated in the FSC/SSC scatter plot. $\mathrm{CD} 4{ }^{+}$lymphocytes were then selected from this gate and analyzed for FOXP3 staining. The negative threshold was set using fluorescence minus one controls (FMO). Duplicate independent experiments were performed for each patient.

\section{ELISA}

Cytokines were quantified in organ culture and LPTC supernatants (each sample was tested in duplicate), following manufacturer's instructions: human IL-6 (R\&D systems, Minneapolis, MN, United States), human IL-8 (BD, Franklin Lakes, NJ, United States), human IFN- $\gamma$ and human TNF- $\alpha$ (ImmunoTools, Friesoythe, Germany), human IL-13 (Invitrogen, Thermo Fisher Scientific, Rockford, IL, United States), human IL-10 (R\&D systems, Minneapolis, MN, United States), human IL-1 $\beta$ and human IL-17 A (Biolegend, San Diego, CA, United States).

\section{Western Blot}

Protein extracts from LPTC incubated with anti-CD3/anti-CD28 alone or combined with L. kefiri for $30 \mathrm{~min}$ were used. Briefly, cells were harvested and lyzed with RIPA buffer (Sigma-Aldrich, St. Louis, MO, United States) in the presence of a protease inhibitor mixture (Sigma-Aldrich, St. Louis, MO, United States). Protein content was quantified by bicinchoninic acid assay (Pierce, Thermo Fisher Scientific, Rockford, IL, United States) and extracts were stored at $-80^{\circ} \mathrm{C}$ until use. Protein samples were resolved on 10\% SDS-PAGE under reducing conditions (BioRad Mini-Protean III; BioRad, Hercules, CA, United States), and transferred onto a nitrocellulose membrane for $1 \mathrm{~h}$ at $300 \mathrm{~mA}$. Blots were blocked and probed with a rabbit anti-p65 antibody (Santa Cruz Biotechnology, Santa Cruz, CA, United States), followed by the appropriate HRP-conjugated secondary antibody (BioRad, Hercules, CA, United States). Protein bands were visualized by enhanced chemiluminescence (ECL Plus; GE Healthcare, Danderyd, Sweden) according to the manufacturer's instructions. Blots were stripped and incubated with a rabbit anti-human $\beta$-actin antibody (Abcam, Cambridge, MA, United States) diluted 1:3,000, as an internal loading control. The bands were scanned with $\mathrm{C}$ digit scanner (LI-COR Biosciences, Lincoln, Nebraska, United States) and quantified using ImageJ software.

\section{Statistical Analysis}

Statistical analysis was carried out using GraphPad Prism eight software (GraphPad Software, San Diego, CA, United States). The significance of the difference was determined using an independent-sample $t$-test or 1-way ANOVA after visual inspections of distribution using Q-Q plots and Shapiro-Wilk normality test analysis. In cases when data did not adjust to normal distribution, Wilcoxon matched paired test or Friedman statistics were applied. A $p$-value $<0.05$ was considered statistically significant. 

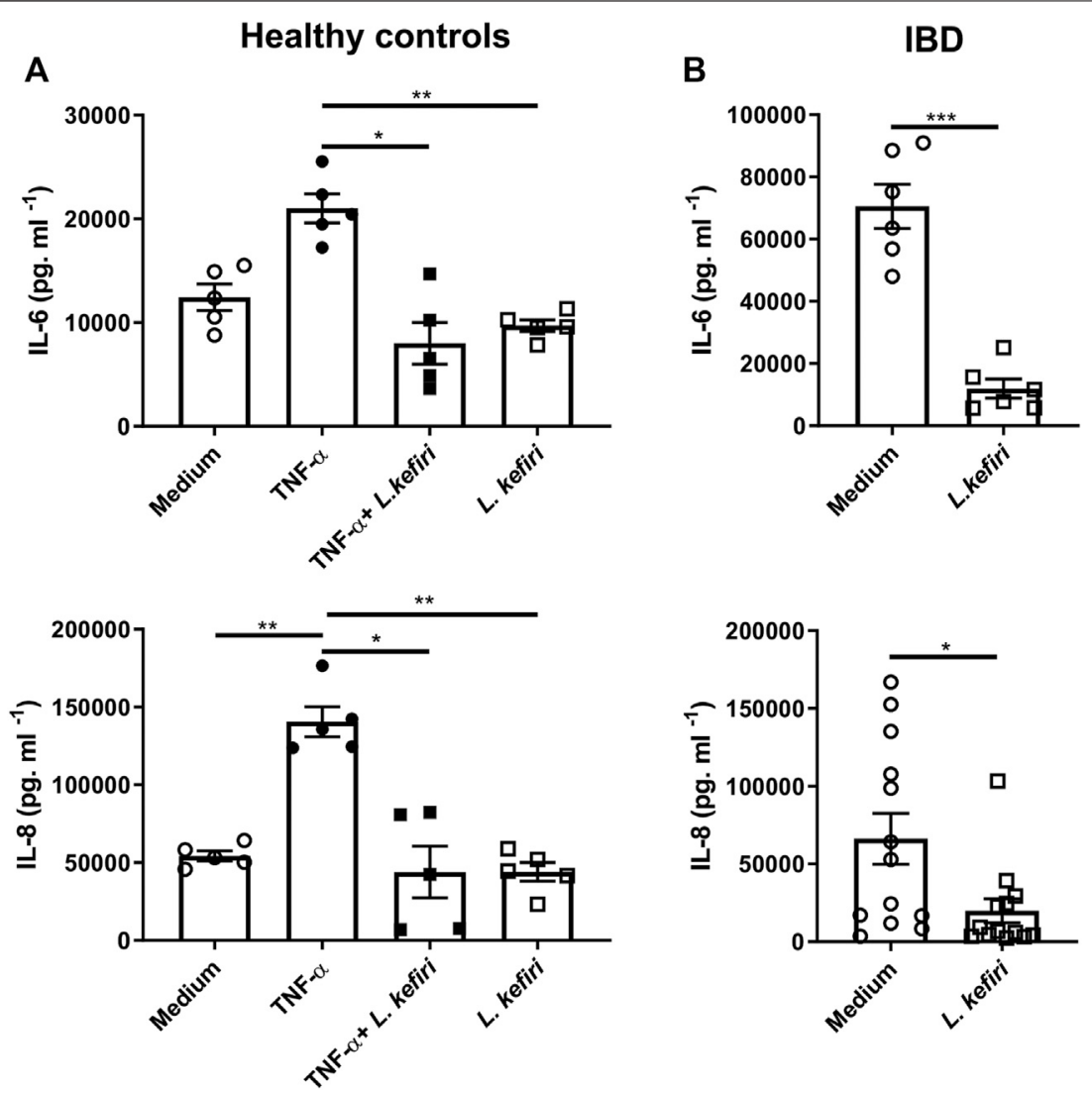

FIGURE 1 | Lactobacillus kefiri modulates the secretion of IL-6 and IL-8 in organ culture (A). Biopsies from healthy donors $(n=5)$ were incubated o. $\mathrm{n}$. with TNF- $\alpha, L$. kefiri or a combination of both (B). Biopsies form IBD patients $(n=13)$ were incubated with or without $L$. kefiri. Supernatants were collected and cytokines were assessed by ELISA. ${ }^{\star} p<0.05,{ }^{\star \star} p<0.01,{ }^{\star \star \star} p<0.001$.

\section{Ethics Statement}

The studies involving human participants were reviewed and approved by Ethics Committee of the Hospital de Gastroenterología Carlos B. Udaondo. The patients/participants provided their written informed consent to participate in this study.

\section{RESULTS}

\section{Lactobacillus Kefiri Reduces the Pro-Inflammatory Cytokine Secretion in Inflamed Biopsies from IBD Patients}

In order to evaluate the immunomodulatory effects of $L$. kefiri CIDCA 8348, we assayed IL- 6 and IL-8 in culture supernatants of tissue explants from healthy subjects $(n=5)$ incubated with the pro-inflammatory stimulus TNF- $\alpha$, with or without probiotics (Figure 1A). TNF- $\alpha$ promoted the secretion of IL-8 $(p<0.01)$, and a trend in IL-6 secretion $(p=0.0616)$ with respect to unstimulated healthy tissue. Explants exposed to L. kefiri produced reduced levels of these TNF- $\alpha$-induced cytokines $(p<$ $0.05)$. We then studied the effect of the bacteria on biopsies from inflamed mucosa of IBD patients $(n=13)$. L. kefiri significantly dampened the spontaneous release of pro-inflammatory cytokines ex vivo $(p<0.001)$ for IL-6 and IL-8 $(p<0.05)$ (Figure 1B). A significant similar suppression of IL-1 $\beta$ and IL-17 A secretion was observed in IBD samples $(p<0.01)$ (Supplementary Figure S1).

\section{Lactobacillus Kefiri Modulates the Cell Proliferation of Stimulated Microbiota-Specific Lamina Propria T Lymphocytes}

Since EA E. coli is overrepresented in the microbiota of IBD patients, we expanded $E$ coli-specific LPTC. We could not retrieve LPTC from HC since these cells did not survive long in vitro under EA E. coli extract stimulation. We therefore proceeded to generate LPTC from 17 colon samples from patients with active IBD. We found that EA $E$. coli extracts significantly increased $(p<$ 0.01 ) the proliferation of LPTC from IBD patients (Figure 2A).

Aiming to study whether L. kefiri modulates IBD LPTC, anti$\mathrm{CD} 3 / \mathrm{CD} 28$-stimulated cells were co-incubated with the probiotics. We found that the increased proliferation of LPTC $(p<0.01)$ and $\mathrm{CD} 4^{+}$LPTC $(p<0.001)$ (Figures 2A-C) were significantly inhibited by incubation with L. kefiri $(p<0.01$ and $p<0.05$, respectively). Also, the cell viability remained unchanged in all conditions, as shown with 7AAD staining (Figure 2D). E. coli used as control promoted cell proliferation, thus 

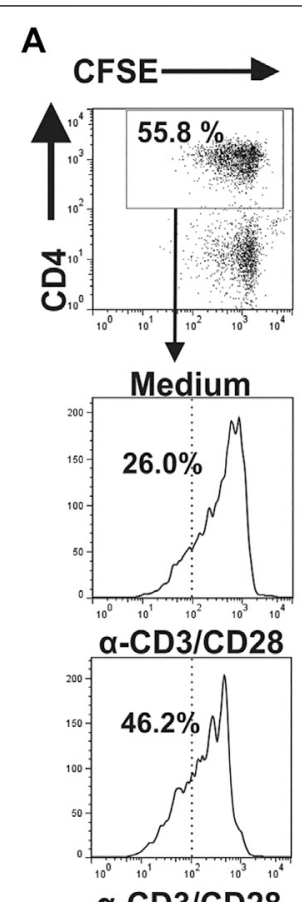

a-CD3/CD28

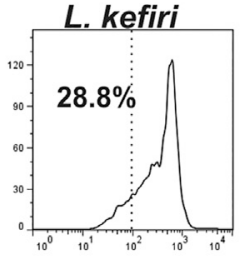

$\alpha-C D 3 / C D 28 \mathrm{CM}$
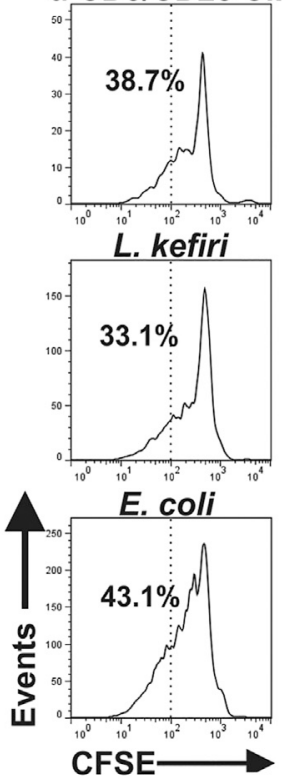

B

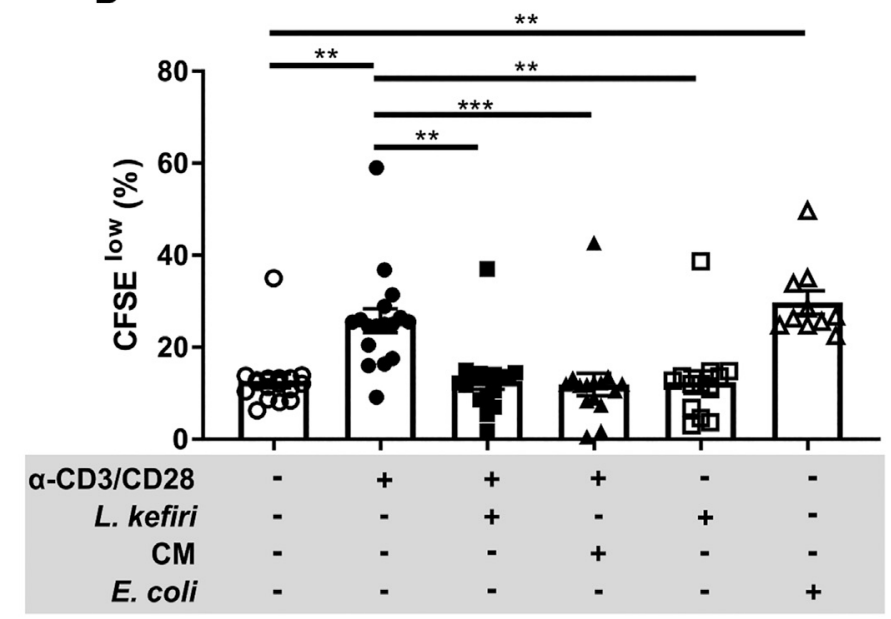

C
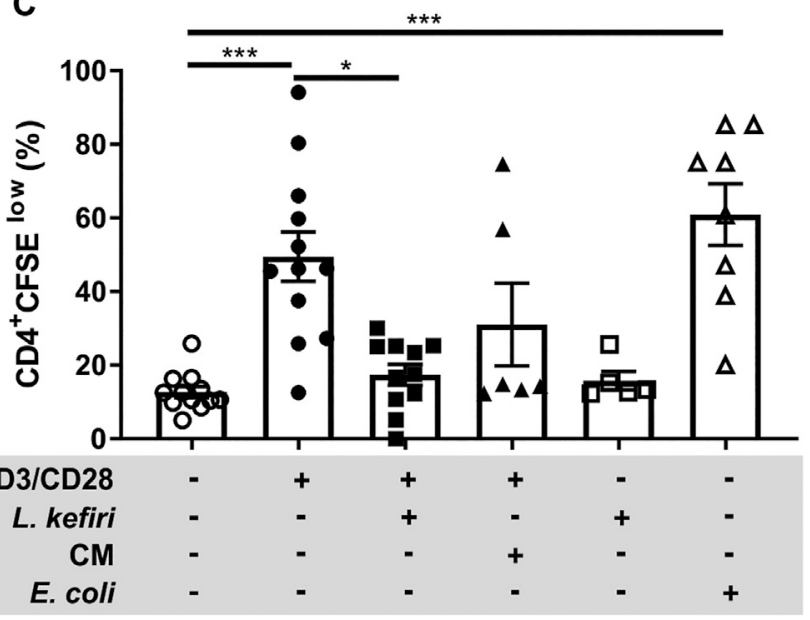

D

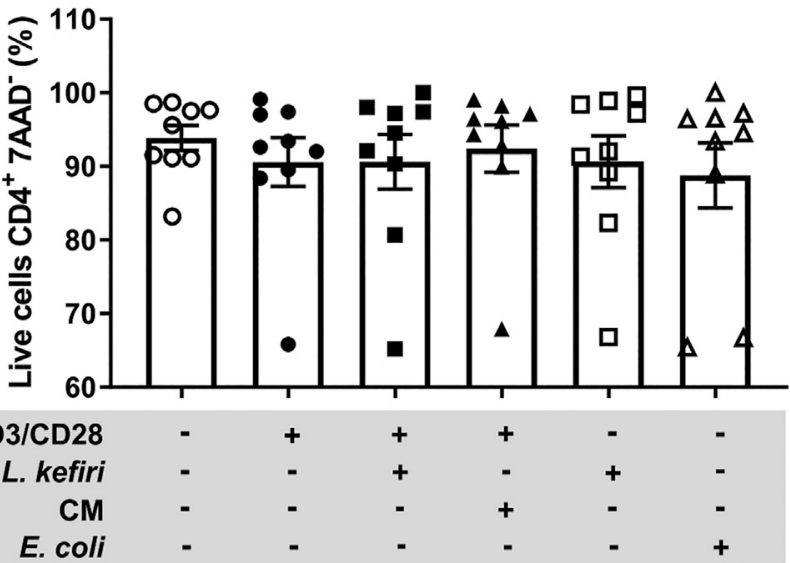

FIGURE 2 | Proliferation and LPTC response are impaired by L. kefiri (A). LPTC were activated with anti-CD3 and anti-CD28 and incubated with live probiotic, conditioned medium (CM), EA E. coli or medium. Cell proliferation was assessed by flow cytometry with CFSE. The gating strategy is shown: CD4 ${ }^{+}$cells were gated from the lymphocyte gate on the forward and side scatter plot. Histograms for CFSE staining are shown separately for each stimulus. Representative results from one patient are shown (B). LPTC proliferation shown as percentage of "CFSE low" staining population under each stimulus in vitro. Each symbol represents one independent experiment (C). Proliferation of CD4 ${ }^{+}$LPTC is shown as percentage of CFSE low cells obtained by the strategy shown in A, each symbol represents one independent experiment (D). CD4 ${ }^{+}$LPTC cell death was assessed by flow cytometry with 7 -AAD staining, in the same LPTC cultures. ${ }^{\star} p<0.05$, ${ }^{\star \star} p<0.01,{ }^{\star \star \star} p<0.001$. 

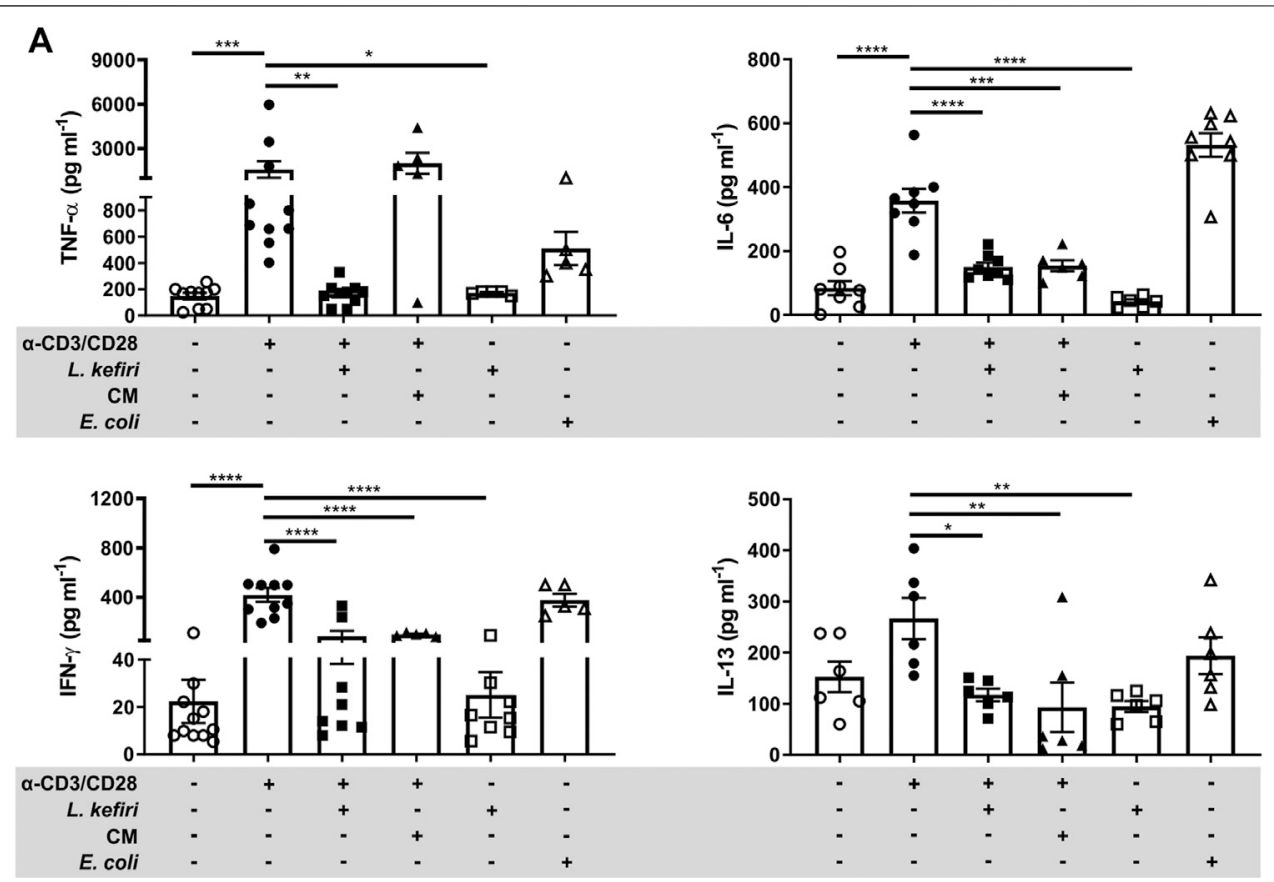

B
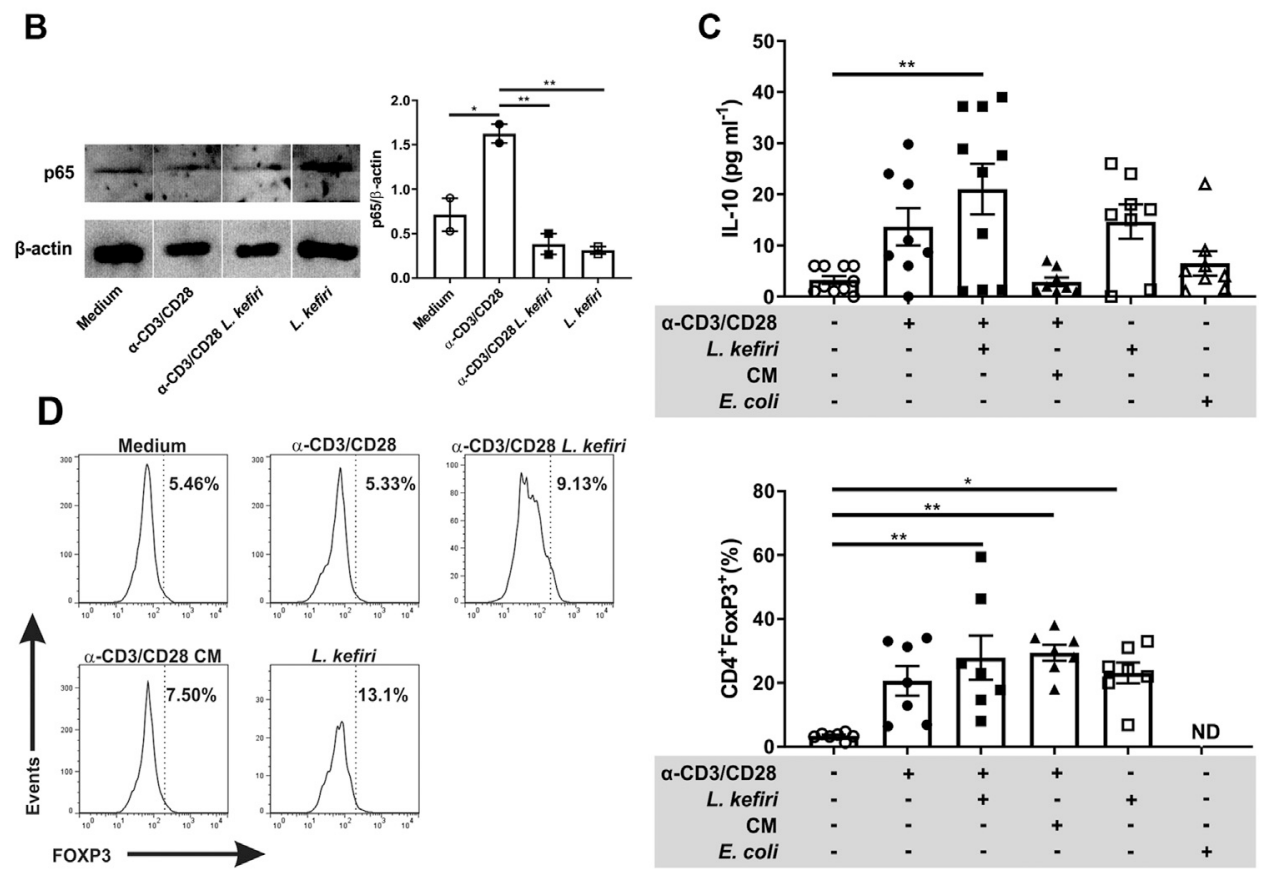

FIGURE 3 | Lactobacillus kefiri modulates LPTC cytokine secretion, FOXP3 expression and NF-kB signaling (A). Effect of L. kefiri and CM on cytokine secretion from activated LPTC. Cytokines were assessed by ELISA (B). Immunoblots of LPTC protein extracts after 30 min of stimulation (representative of two independent assays) and statistical analysis of intensities of bands corresponding to p65 (C). Quantification of IL-10 by ELISA in supernatants of the same LPTC assays shown in A (D). Representative histograms of the flow cytometry data analysis of LPTC. Frequency of CD4 ${ }^{+} \mathrm{FOXP3}{ }^{+} \mathrm{T}$ cells after 4 days incubation with live probiotic or CM. $\mathrm{FOXP3}^{+}$cells were evaluated in CD4 ${ }^{+}$cells from the lymphocytes gate. Anti-CD3 and anti-CD28 antibodies were used for T cell activation. ${ }^{*} p<0.05,{ }^{\star \star} p<0.01$, ${ }^{\star \star \star} p<0.001,{ }^{\star \star \star \star} p<0.0001$.

confirming the specificity of T cells $(p<0.01$ and $p<0.001$, respectively).

Considering that lactic acid bacteria secrete SCFA with immunomodulatory properties, we also incubated LPTC with conditioned medium from L. kefiri. As depicted, we found that LPTC proliferation was significantly suppressed $(p<0.001)$, whereas for activated-CD $4^{+} \mathrm{T}$ cells, it did not reach statistical significance $(p=0.106)$ (Figures 2A-D). 


\section{Lactobacillus Kefiri Ameliorates the Pro-Inflammatory Cytokine Secretion by Stimulated-Microbiota-Specific Lamina Propria T Cells}

To further characterize the cellular response of these cells in vitro, we evaluated the secretion of pro-inflammatory cytokines by activated LPTC when co-incubated with $L$. kefiri. The stimulation of LPTC with anti-CD3/CD28 induced the secretion of TNF- $\alpha$, IFN- $\gamma$, IL- 6 and IL-13. The co-incubation of activated LPTC with the probiotic significantly diminished the secretion of these cytokines (Figure 3A, $p<0.01, p<0.0001, p<0.0001$ and $p<$ 0.05 respectively). Also, the incubation of the stimulated cells with CM significantly reduced the levels of the pro-inflammatory cytokines IFN- $\gamma(p<0.0001)$, IL-6 $(p<0.001)$ and IL-13 $(p<0.01)$.

We then investigated the activation of the NF- $\kappa B$ pathway on LPTC from patients with active IBD in the different culture conditions. We found that $\mathrm{p} 65$ levels were diminished in activated cells after a $30 \mathrm{~min}$ exposure to L. kefiri (Figure 3B, $p<0.01$ ). $L$. kefiri per se did not trigger the NF- $\mathrm{kB}$ pathway.

Next, we analyzed the secretion of the tolerogenic cytokine IL-10 and we found it significantly increased in the supernatant of stimulated LPTC that were exposed to L. kefiri (Figure 3C, $p$ $<0.01$ ). To get a further insight into this suppressive effect, FOXP3 expression was also evaluated in LPTC by flow cytometry (Figure 3D). We found that this transcription factor was specifically induced in $\mathrm{CD}^{+}$LPTC by L. kefiri, irrespective of their activation with anti-CD3/anti-CD28 $(p<0.05)$. We also found that CM induced FOXP3 expression in activated LPTC $(p<0.01)$ (Figure 3D).

\section{DISCUSSION}

In IBD, dysregulated immune responses take place against the intestinal microbiota in genetically predisposed hosts, and several $\mathrm{T}$ cell subsets have been described to be involved in homeostasis breakdown. In this context, microbiota-specific $\mathrm{T}$ cells were identified in IBD patients and in animal models (Hepworth et al., 2015; Sorini et al., 2018). Intestinal T cells are target of several therapeutic procedures to constrain inflammation.

In our study, we aimed to evaluate the immunomodulatory properties of a probiotic Lactobacillus strain isolated from kefir on lamina propria $\mathrm{CD} 4^{+} \mathrm{T}$ cells from patients with active IBD. Microbiota-specific lamina propria $\mathrm{T}$ cell lines were obtained from intestinal specimens and exposed to L. kefiri CIDCA 8348 or conditioned medium to mitigate cell activation. The control of cell proliferation and cytokine secretion of activated $\mathrm{T}$ cells correlated significantly with previous results from our group found in healthy mice and in mice fed with a fructose-rich diet that were orally administered with this bacteria (P. Carasi et al., 2015; Zubiría et al., 2017).

Probiotic microorganisms isolated from kefir as well as some of their metabolites have been reported to reduce gut inflammation in colitis animal models, and the amelioration of symptoms after kefir consumption has been observed in one controlled trial with IBD patients to date (Iraporda et al., 2016; Sevencan et al., 2019; Y1lmaz, Dolar, and Özpinar 2019). However, this is the first report to show the immunomodulation promoted by Lactobacillus kefiri in human organ culture ex vivo. The patients in our study were on immunosuppressive and/or immunomodulatory pharmacological treatment, and we considered this could be interfering with our ex vivo model results. However, mucosal biopsies obtained from inflamed colon areas of IBD patients showed increased basal levels of pro-inflammatory cytokines IL-6, IL- 8 and even IL-1 $\beta$ and IL-17A, reflecting the periods of flares and increased disease activity occurring also in treated patients. We showed that $L$. kefiri CIDCA 8348 suppressed the secretion of these pro-inflammatory cytokines from IBD mucosal biopsies to similar levels as those found in healthy mucosa. Similar results were recently reported in a study performed by Pagnini et al., with a dose-response reduction of TNF- $\alpha$ and IL-17 expression in UC mucosal samples incubated with $L$. rhamnosus GG (Pagnini et al., 2018). In another study, IBD biopsies exposed to the probiotic Lactococcus lactis exhibited a reduced secretion of TNF- $\alpha$ and IL-23 (Simčič et al., 2019).

Considering that the gut barrier is impaired in IBD and luminal microorganisms may be found in the lamina propria of patients, we further addressed the effect of $L$. kefiri on lamina propria activated microbiota-specific $T$ cells, to deeper understand whether L. kefiri's anti-inflammatory effect occurred through lamina propria T lymphocyte modulation (Kumar et al., 2020; Al-Sadi et al., 2021). We isolated lamina propria mononuclear cells and established entero-adhesive E. coli-specific T cell lines for in vitro characterization. LPTC were activated with anti-CD3 and antiCD28 antibodies, and we found that cell proliferation, cytokine secretion and NF- $\kappa \mathrm{B}$ pathway activation were suppressed upon exposure to L. kefiri. Collectively, different strains of Lactobacillus and Lactococcus have shown to modulate the inflammatory activity through multiple mechanisms, but especially by inhibiting the translocation of the nuclear transcription factor NF- $\mathrm{B}$ (Vincenzi et al.,. 2020; Zeng et al., 2020). Here, we co-incubated activated LPTC in vitro with $L$. kefiri and we found that NF- $\kappa \mathrm{B}$ p 65 was diminished in these cells, which also secreted lower amounts of TNF- $\alpha$, IFN- $\gamma$, IL- 6 and IL- 13 than controls. In addition, activated LPTC incubated with L. kefiri CM also exhibited a reduced secretion of these cytokines. However, as proliferation of $\mathrm{CD}^{+}$ $\mathrm{T}$ cells from these LPTC cultures was not diminished by L. kefiri CM, we propose that L. kefiri effect might be due to a direct interaction between the bacteria and the $\mathrm{CD} 4^{+} \mathrm{T}$ lymphocytes. To this regard, it has been previously described that the purified S-layer glycoprotein of L. kefiri CIDCA 8348 (a regular protein array that completely covers the bacterial surface) could enhance the activation of murine macrophages and bone marrow derived dendritic cells, through the recognition of the S-layer protein glycans by the C-type lectin receptor Mincle (Malamud et al., 2019). However, further investigations are needed to understand the role of the S-layer protein recognition on tuning the activation of antigen-presenting cells triggered by the whole bacteria. Nonetheless, a recent report by Gong et al. showed increased Mincle signaling in both intestinal samples from CD patients and experimental models of colitis, mainly due to an up-regulation of pyroptosis in macrophages, which promotes gut inflammation (Gong et al., 2020). However, the expression of Mincle on human $\mathrm{CD}^{+} \mathrm{T}$ lymphocytes has not been widely reported (Vijayan et al. 2010). Therefore the level of expression in LPTC from IBD patients 
and the possible role of this $\mathrm{C}$-type lectin receptor in the interaction between LPTC CD4 ${ }^{+}$and L. kefiri CIDCA 8348 will be key issues to be addressed in further studies. The effect of conditioned medium on cytokine secretion may be attributed to metabolites secreted by lactobacilli. Of note, we have measured lactate present in L. kefiri conditioned medium used for the assay and it was $140 \pm 0.2 \mu \mathrm{M}$. We have performed proliferation and cytokine assays including lactate as a possible modulator (Garrote et al., 2015; Iraporda et al., 2016), but results have been significant for concentrations above $10 \mathrm{mM}$ (data not shown), a value much higher than that secreted by cultured L. kefiri. Hence the effect shown in this work cannot be attributed solely to this SCFA and must be further investigated.

Remarkably, in our study we found that LPTC incubated with L. kefiri or conditioned medium showed a high frequency of $\mathrm{CD} 4^{+}$ FOXP3 $^{+} \mathrm{T}$ cells. Several studies highlight that Lactobacillus may promote Treg differentiation in animal models (Zakostelska et al., 2011; Smelt et al., 2013; Park et al., 2017). Long term Treg cultures, like the ones performed in this work, have been shown to lose FOXP3 signaling upon repeated stimulation cycles (Hoffmann et al., 2009). In mice, in vitro incubation of $\mathrm{CD}^{+} \mathrm{T}$ cells with sonicated extracts of Lactobacillus rhamnosus GG diminished IL17 secretion by these cells and increased FOXP3 and IL-10 secretion via TLR2 mechanism, clearly showing the plasticity induced by the probiotic (Jia et al., 2020). Our results could be showing the functional plasticity of human lamina propria effector $\mathrm{T}$ cells in response to the probiotic or its metabolites, which may induce FOXP3 induction. Our experiments showed a direct effect of L. kefiri on T cell IL-10 secretion, since no antigen presenting cells were included in our in vitro assays. Further studies are needed to characterize whether the $\mathrm{FOXP3}^{+}$cells are responsible for the increase in IL-10 secretion, and functional assays should be performed to demonstrate the regulatory capacity of these $\mathrm{CD} 4^{+}$ $\mathrm{FOXP}^{+} \mathrm{T}$ cells in the future. It is worth noting that the increase of IL-10 secretion induced by the probiotic may be important for generating a tolerogenic milieu in the inflamed gut, even in the absence of FOXP3 induction (Hoffmann et al., 2009). These effects, combined with a lower proliferation of effector $\mathrm{CD}^{+} \mathrm{T}$ lymphocytes and the decrease in pro-inflammatory cytokine secretion could be a useful complement of the adequate drug treatment to shift the IBD gut toward a tolerogenic state.

Although the anti-inflammatory activity of L. kefiri CIDCA 8348 has been shown in mice (Carasi et al., 2015; Zubiría et al., 2017), this is the first report demonstrating the immunomodulatory properties of a kefir-isolated strain (L. kefiri CIDCA 8348) on human intestinal tissue and primary T cells from IBD patients. Great effort has been made to modulate the pro-inflammatory activity of $\mathrm{T}$ cells as a treatment for IBD, with variable success; however, there is still need for improving therapies. Understanding the mechanisms of interaction between probiotics and immune cells in the gut could open new avenues to help prevent or treat inflammatory bowel disease.

\section{DATA AVAILABILITY STATEMENT}

The original contributions presented in the study are included in the article/Supplementary Material, further inquiries can be directed to the corresponding author.

\section{ETHICS STATEMENT}

The studies involving human participants were reviewed and approved by Comité de Ética e Investigaciones, Hospital de Gastroenterología Dr. Carlos Bonorino Udaondo Av Caseros 2,153, 3er Piso 1,264 Ciudad de Buenos Aires. The patients/ participants provided their written informed consent to participate in this study.

\section{AUTHOR CONTRIBUTIONS}

All authors have made substantial contributions to the study. Conception and design of the study: CM and MS. Acquisition of data: RC, CM, KC, IS, EBR. Intestinal biopsies or surgical pieces sampling: AR, ID, EP, SB, GC, MY and LG. Patients recruitment and clinical diagnosis: AS, SG, PT, GC and MY. Analysis and/or interpretation of data: CM, MS, GD, RC and KC. Drafting the manuscript: CM, MS, RC. Revising the manuscript critically for important intellectual content: CM, MS, GD, RC, KC, and AS. Approval of the version of the manuscript to be published: CM, MS, GD, RC, KC, IS, EBR, AR, ID, EP, SB, GC, MY, LG, AS.

\section{FUNDING}

This research was supported by Grants from the Agencia Nacional de Promoción Científica y Técnológica (PICT 20162387 to RC, PICT 2016-0648 to CIM, PICT 2016-0244 to MAS, PICT 2015-1648 to GHD). All grants are for research materials. All funders contributed equally to this work. RC, GHD, MAS and CIM are researchers from Consejo Nacional de Investigaciones Científicas y Técnicas of Argentina (CONICET); KEC and EBR are postdoctoral and doctoral fellows from CONICET, respectively.

\section{ACKNOWLEDGMENTS}

We thank Dr. Ayelén Hugo for providing entero-adhesive E. coli cultures and Dr. Martín Rumbo for his support and advice. We are very grateful to the staff at the endoscopy unit at Hospital Udaondo, as well as to all patients who kindly contributed to this study. We thank the technician staff from the FACS facilities and cell and tissue culture lab at IIFP for their help and contribution to this research. CM also wants to thank Professor Dr. Barbara Bohle, for kindly sharing her knowledge on the establishment of $\mathrm{T}$ cell lines. We also thank Dr. Anna Vossenkaemper for her critical reading of the manuscript.

\section{SUPPLEMENTARY MATERIAL}

The Supplementary Material for this article can be found online at: https:/www.frontiersin.org/articles/10.3389/fphar.2021.658026/ full\#supplementary-material. 


\section{REFERENCES}

Abdelhalim, K. A., Uzel, A., and Gülşen Ünal, N. (2020). Virulence determinants and genetic diversity of adherent-invasive Escherichia coli (AIEC) strains isolated from patients with Crohn's disease'Virulence determinants and genetic diversity of adherent-invasive Escherichia coli (AIEC) strains isolated from patients with crohn's disease'. Microb. Pathogenesis 145, 104233. doi:10. 1016/j.micpath.2020.104233

Ahn, S.-I., Cho, S., and Choi, N.-J. (2020). Effect of dietary probiotics on colon length in an inflammatory bowel disease-induced murine model: a metaanalysis. J. Dairy Sci. 103 (2), 1807-1819. doi:10.3168/jds.2019-17356

Al-Sadi, R., Nighot, P., Nighot, M., Haque, M., Rawat, M., Ma, T. Y., et al. (2021). Lactobacillus acidophilus induces a strain-specific and toll-like receptor-2 dependent enhancement of intestinal epithelial tight junction barrier and protection against intestinal inflammation. Am. J. Pathol. S0002-9440, 48-112. doi:10.1016/j.ajpath.2021.02.003

Bengoa, A. A., Iraporda, C., Garrote, G. L., and Abraham, A. G. (2019). Kefir microorganisms: their role in grain assembly and health properties of fermented milk. J. Appl. Microbiol. 126 (3), 686-700. doi:10.1111/jam.14107

Bohle, B., Radakovics, A., Jahn-Schmid, B., Hoffmann-Sommergruber, K., Fischer, G. F., and Ebner, C. (2003). Bet v 1, the major birch pollen allergen, initiates sensitization to api $\mathrm{g} 1$, the major allergen in celery: evidence at the $\mathrm{T}$ cell level. Eur. J. Immunol. 33 (12), 3303-3310. doi:10.1002/eji.200324321

Carasi, P., Racedo, S. M., Jacquot, C., Romanin, D. E., Serradell, M. A., and Urdaci, M. C. (2015). Impact of kefir derived Lactobacillus kefiri on the mucosal immune response and gut microbiota. J. Immunol. Res. 2015, 361604. doi:10. $1155 / 2015 / 361604$

Carasi, Paula., Díaz, Mariángeles., and Silvia, M. (2014). Safety characterization and antimicrobial properties of kefir-isolated Lactobacillus Kefiri. Biomed. Res. Int. 2014, 208974 doi:10.1155/2014/208974

Fan, H., Du, J., Du, J., Liu, X., Zheng, W.-W., Zhuang, Z.-H., et al. (2019). Effects of pentasa-combined probiotics on the microflora structure and prognosis of patients with inflammatory bowel disease. Turk J. Gastroenterol. 30 (8), 680-685. doi:10.5152/tjg.2019.18426

Farag, M. A., Jomaa, S. A., El-Wahed, A. A., and El-Seedi, H. R.. (2020). The many faces of kefir fermented dairy products: quality characteristics, flavour chemistry, nutritional value, health benefits, and safety. Nutrients 12 (2), 112. doi:10.3390/nu12020346

Food and Agriculture Organization and of the United Nations/World Health Organization. (2002). Guidelines for the evaluation of probiotics in foods, Available at: https://www.who.int $>$ fs_management $>$ probiotic_guidelines.

Garrote, G. L., Serradell, M. A, Abraham, A. G., Añon, M. C., Fossati, C. A., and De Antoni, G. L. (2005). Development of an immunochemical method to detectLactobacillus kefir. Food Agric. Immunol. 16 (3), 221-233. doi:10. 1080/09540100500244146

Garrote, G. L., Abraham, A. G., and Martín, R. (2015). Is lactate an undervalued functional component of fermented food products?. Front. Microbiol. 6, 629. doi:10.3389/fmicb.2015.00629

Gong, W., Zheng, T., Guo, K., Miao, F., Xie, H., Li, W., et al. (2020). Mincle/Syk signaling promotes intestinal mucosal inflammation through induction of macrophage pyroptosis in crohn's disease. J. Crohn's Colitis 25, jjaa088. doi:10.1093/ecco-jcc/jjaa088

Hepworth, M. R., Fung, T. C., Masur, S H., Kelsen, J. R., McConnell, F. M., Dubrot, J., et al. (2015). Group 3 innate lymphoid cells mediate intestinal selection of commensal bacteria-specific CD4+ T cells. Science 348 (6238), 1031-1035. doi:10.1126/science.aaa4812

Hoffmann, P., Boeld, T. J., Eder, R., Huehn, J., Floess, S., Wieczorek, G., et al. (2009). Loss of FOXP3 expression in natural human CD4+CD25+regulatory $\mathrm{T}$ cells upon repetitivein vitrostimulation. Eur. J. Immunol. 39 (4), 1088-1097. doi:10.1002/eji.200838904

Iraporda, C., Romanin, D, E., Bengoa, A. A., Errea, J. A., Cayet, D., Foligné, B, et al. (2016). Local treatment with lactate prevents intestinal inflammation in the TNBS-induced colitis model. Front. Immunol. 7, 651. doi:10.3389/fimmu.2016. 00651

Jakubczyk, D., Leszczyńska, K., and Górska, S. (2020). The effectiveness of probiotics in the treatment of inflammatory bowel disease (IBD)-A critical review. Nutrients 12 (7). doi:10.3390/nu12071973
Jia, L., Wu, R., Han, N., Fu, J., Luo, Z., Guo, L., et al. (2020). Porphyromonas gingivalis and Lactobacillus rhamnosus GG regulate the Th17/treg balance in colitis via TLR4 and TLR2. Clin. Transl. Immunol. 9 (11), e1213. doi:10.1002/ cti2.1213

Kaser, A., and Blumberg, R. S. (2011). Autophagy, microbial sensing, endoplasmic reticulum stress, and epithelial function in inflammatory bowel disease. Gastroenterology 140 (6), 1738-1747. doi:10.1053/j.gastro.2011.02.048

Kumar, M., Leon Coria, A., Cornick, S., Petri, B., Mayengbam, S., Jijon, H. B., et al. (2020). Increased intestinal permeability exacerbates sepsis through reduced hepatic SCD-1 activity and dysregulated iron recycling. Nat. Commun. 11 (1), 483. doi:10.1038/s41467-019-14182-2

Lee, J. G., Dong, S. H., Su, V. J., Reum, L., Chan Hyuk, P., Eun, C. S., et al. (2019). Characteristics and pathogenic role of adherent-invasive Escherichia coli in inflammatory bowel disease: potential impact on clinical outcomes. PLoS ONE 14 (4), e0216165. doi:10.1371/journal.pone.0216165

Lee, J. S., Wang, R. X., Alexeev, E. E., and Colgan, S. P. (2021). Intestinal inflammation as a dysbiosis of energy procurement: new insights into an old topic. Gut Microbes 13 (1), 1-20. doi:10.1080/19490976.2021.1880241

Liu, S., Zhao, W., Lan, P., and Mou, X. (2020). 'The microbiome in inflammatory bowel diseases: from pathogenesis to therapy'. Protein Cell 12, 33. doi:10.1007/ s13238-020-00745-3

Malamud, M., Carasi, P., Assandri, M. H., Freire, T., Lepenies, B., and Serradell, M. D. L. Á.. (2019). S-layer glycoprotein from Lactobacillus kefiri exerts its immunostimulatory activity through glycan recognition by Mincle. Front. Immunol. 10, 1422. doi:10.3389/fimmu.2019.01422

Onderdonk, A. B., Hermos, J. A., and Bartlett, J. G. (1977). The role of the intestinal microflora in experimental colitis. Am. J. Clin. Nutr. 30 (11), 1819-1825. doi:10. 1093/ajcn/30.11.1819

Pagnini, C., Corleto, V. D., Martorelli, M., Lanini, C., D’Ambra, G., Giulio, E. D., et al. (2018). Mucosal adhesion and anti-inflammatory effects ofLactobacillus rhamnosusGG in the human colonic mucosa: a proof-of-concept study. WJG 24 (41), 4652-4662. doi:10.3748/wjg.v24.i41.4652

Park, J.-S., Joe, I., Rhee, P. D, Jeong, C-S., and Jeong, G. (2017). A lactic acid bacterium isolated from kimchi ameliorates intestinal inflammation in DSSinduced colitis. J. Microbiol. 55 (4), 304-310. doi:10.1007/s12275-017-6447-y

Peyrin-Biroulet, L., Panés, J., Sandborn, W. J., Vermeire, S., Danese, S., Feagan, B. G., et al. (2016). Defining disease severity in inflammatory bowel diseases: current and future directions. Clin. Gastroenterol. Hepatol. 14 (3), 348-354. doi:10.1016/j.cgh.2015.06.001

Postler, T. S., Ghosh, S., and Ghosh, S. (2017). Understanding the holobiont: how microbial metabolites affect human health and shape the immune system. Cel Metab. 26 (1), 110-130. doi:10.1016/j.cmet.2017.05.008

Qiao, Y. Q., Cai, C. W., and Ran, Z. H. (2016). Therapeutic modulation of gut microbiota in inflammatory bowel disease: more questions to Be answered. J. Dig. Dis. 17 (12), 800-810. doi:10.1111/1751-2980.12422

Rabinowitz, K. M., Wang, Y, Chen, E. Y., Hovhannisyan, Z., Chiang, D., Berin, M. C., et al. (2013). Transforming growth factor $\beta$ signaling controls activities of human intestinal CD8+T suppressor cells. Gastroenterology 144 (3), 601-612. doi:10.1053/j.gastro.2012.12.001

Schlegel, N., Boerner, K., and Waschke, J. (2020). Targeting desmosomal adhesion and signalling for intestinal barrier stabilization in inflammatory bowel diseases-lessons from experimental models and patients, Acta physiologica 231. e13492. doi:10.1111/apha.13492

Schmitt, H., Neufert, C., Neurath, M. F., and Atreya, R. (2019). Resolution of Crohn's disease. Semin. Immunopathol 41 (6), 737-746. doi:10.1007/s00281019-00756-1

Sevencan, N. O., Isler, M., Kapucuoglu, F. N., Senol, A., Kayhan, B., Kiztanir, S., et al. (2019). Dose-dependent effects of kefir on colitis induced by trinitrobenzene sulfonic acid in rats. Food Sci. Nutr. 7 (9), 3110-3118. doi:10.1002/fsn3.1174

Shanahan, F., and Quigley, E. M. M. (2014). Manipulation of the microbiota for treatment of IBS and IBD-challenges and controversies. Gastroenterology 146 (6), 1554-1563. doi:10.1053/j.gastro.2014.01.050

Shawki, A., and McCole, D. F. (2017). Mechanisms of intestinal epithelial barrier dysfunction by adherent-invasive Escherichia coli. Cell Mol. Gastroenterol. Hepatol. 3 (1), 41-50. doi:10.1016/j.jcmgh.2016.10.004

Simčič, S., Berlec, A., Stopinšek, S., Štrukelj, B., and Orel, R. (2019). 'Engineered and wild-type L. Lactis promote anti-inflammatory cytokine signalling in 
inflammatory bowel disease patient's mucosa'. World J. Microbiol. Biotechnol. 35 (3), 45. doi:10.1007/s11274-019-2615-z

Slattery, C., Cotter, P. D., and O'Toole, P. W. (2019). Analysis of health benefits conferred by Lactobacillus species from kefir. Nutrients 11 (6), 113. doi:10.3390/ nu11061252

Smelt, M. J., de Haan, B. J., Bron, P. A., Iris van, S., Meijerink, M., Wells, J. M., et al. (2013). Probiotics can generate FoxP3 T-cell responses in the small intestine and simultaneously inducing CD4 and CD8 T cell activation in the large intestine. PLoS One 8 (7), e68952. doi:10.1371/journal.pone.0068952

Sorini, C., Cardoso, R. F., Gagliani, N., and Villablanca, E. J. (2018). Commensal bacteria-specific $\mathrm{CD} 4+\mathrm{T}$ cell responses in health and disease. Front. Immunol. 9, 2667. doi:10.3389/fimmu.2018.02667

Torres, J., Mehandru, S., Colombel, J.-F., and Peyrin-Biroulet, L. (2017). Crohn's disease. The Lancet 389 (10080), 1741-1755. doi:10.1016/s0140-6736(16)31711-1

Ungaro, R., Mehandru, S. P., Peyrin-Biroulet, L., Colombel, J.-F., and Colombel, J.-F. (2017). Ulcerative colitis. The Lancet 389 (10080), 1756-1770. doi:10. 1016/s0140-6736(16)32126-2

Vijayan, D., Radford, K., Bellette, B., Beckhouse, A., Ashman, R., Wells, C., et al. (2010). Expression analysis of MINCLE on human peripheral blood cells. IADR General Session 2010

Vincenzi, A., Márcia, I. G., and Fernanda Volken de Souza, C. (2020), An evaluation of the effects of probiotics on tumoral necrosis factor (TNF- $\alpha$ ) signaling and gene expression. Cytok. Growth Factor Rev. 57, 27-38. doi:10. 1016/j.cytogfr.2020.10.004

Wegh, C. A. M., Geerlings, S. Y., Jan, K., Roeselers, G., and Belzer, C. (2019). Postbiotics and their potential applications in early Life nutrition and beyond. Int. J. Mol. Sci. 20 (19). doi:10.3390/ijms20194673

Yanai, H., and Hanauer, S. B. (2011). Assessing response and loss of response to biological therapies in IBD. Am. J. Gastroenterol. 106 (4), 685-698. doi:10.1038/ ajg.2011.103

YuChia-Hui, L. (2018). Microbiota dysbiosis and barrier dysfunction in inflammatory bowel disease and colorectal cancers: exploring a common ground hypothesis. J. Biomed. Sci. 25 (1), 79. doi:10.1186/s12929-018-0483-8
Yılmaz, İ., Dolar, M. E., and Özpınar, H. (2019). Effect of administering kefir on the changes in fecal microbiota and symptoms of inflammatory bowel disease: a randomized controlled trial. Turkish J. Gastroenterol. Official J. Turkish Soc. Gastroenterol. 30 (3), 242-253. doi:10.5152/tjg. 2018.18227

Zakostelska, Z., Kverka, M., Klimesova, K., Pavel, R., Mrazek, J., Jan, K., et al (2011). Lysate of probiotic Lactobacillus casei DN-114 001 ameliorates colitis by strengthening the gut barrier function and changing the gut microenvironment. PLoS One 6 (11), e27961. doi:10.1371/journal.pone.0027961

Zenewicz, L. A., Antov, A., and Flavell, R. A. (2009). CD4 T-cell differentiation and inflammatory bowel disease. Trends Mol. Med. 15 (5), 199-207. doi:10.1016/j. molmed.2009.03.002

Zeng, L., Tan, J., Xue, M., Le, L., Wang, M., Liang, L., et al. (2020). An engineering probiotic producing defensin-5 ameliorating dextran sodium sulfate-induced mice colitis via inhibiting NF-KB pathway. J. Translational Med. 18 (1), 107. doi:10.1186/s12967-020-02272-5

Zubiría, G. M., Gambaro, S. E., María Amanda, R., Carasi, P., María de Los Ángeles, S., and Giovambattista, A. (2017). Deleterious metabolic effects of high fructose intake: the preventive effect of Lactobacillus kefiri administration. Nutrients 9 (5), 72, doi:10.3390/nu9050470

Conflict of Interest: The authors declare that the research was conducted in the absence of any commercial or financial relationships that could be construed as a potential conflict of interest.

Copyright (๑ 2021 Curciarello, Canziani, Salto, Barbiera Romero, Rocca, Doldan, Peton, Brayer, Sambuelli, Goncalves, Tirado, Correa, Yantorno, Garbi, Docena, Serradell and Muglia. This is an open-access article distributed under the terms of the Creative Commons Attribution License (CC BY). The use, distribution or reproduction in other forums is permitted, provided the original author(s) and the copyright owner(s) are credited and that the original publication in this journal is cited, in accordance with accepted academic practice. No use, distribution or reproduction is permitted which does not comply with these terms. 